\title{
Synthesis, spectral studies and biological activity of $3 H$-1, 5-benzodiazepine derivatives
}

\author{
Rajesh Kumar* and Y. C. Joshi \\ Department of Chemistry, University of Rajasthan, Jaipur, 302004 \\ E-mail:rnunia@yahoo.com
}

\begin{abstract}
Chlorination of 5-(2-ethoxyphenyl)-1-methyl-3-propyl-1,6-dihydro-7H-pyrazolo $\quad[4,3-d]$ pyrimidin-7-one (1) with $\mathrm{POCl}_{3}$, afforded 5-(2-ethoxyphenyl)-1-methyl-7-chloro- $1 \mathrm{H}$-pyrazolo $[4,3-d]$ pyrimidine (2). Further, compound 2 condensed with different $\beta$-diketones/ $\beta$-ketoesters 3a-e, to obtain new $\beta$-diketones/ $\beta$-ketoesters 4a-e. These $\beta$-diketones/ $\beta$-ketoesters 4a-e were condensed with $o$-phenylenediamine (o-PDA) to give biologically active $3 H-1,5$ benzodiazepines 5a-e.
\end{abstract}

Keywords- Pyrazolo [4,3- $d$ ] pyrimidin-7-one, $\beta$-diketones/ $\beta$-ketoesters, $p$-toluenesulfonic acid, $o$-phenylenediamine, 1,5-benzodiazepines, antimicrobial and anthelmintic activities

\section{Introduction}

Benzodiazepines have attracted attention as an important class of heterocyclic compounds in the field of drugs and pharmaceuticals. These compounds are widely used as anticonvulsant, antianxiety, analgesic, sedative, anti-depressive, hypnotic agents ${ }^{1}$ as well as anti-inflammatory agents ${ }^{2}$. Other than their biological importance, benzodiazepine derivatives are also commercially used as dyes for acrylic fibres ${ }^{3}$. Moreover, 1,5-benzodiazepines derivatives are valuable synthons that can be used in preparation of other fused ring compounds such as triazolo-, oxadiazolo-, oxazino-,or furano-benzodiazepines ${ }^{4}$. Research in this area is still very active and is directed towards the synthesis of compounds with enhanced pharmacological activity. Generally, these compounds are synthesized by the condensation of $o$-phenylenediamines with $\alpha, \beta$-unsaturated carbonyl compounds ${ }^{5}, \beta$-haloketones, or ketones ${ }^{6}$. A variety of reagents, such as $\mathrm{BF}_{3}$-etherate, $\mathrm{NaBH}_{4}$, polyphosphoric acid, or $\mathrm{SiO}_{2}, \mathrm{MgO} / \mathrm{POCl}_{3}, \mathrm{Yb}(\mathrm{OTf})_{3}, \mathrm{Sc}(\mathrm{OTf})_{3}, \mathrm{Al}_{2} \mathrm{O}_{3} / \mathrm{P}_{2} \mathrm{O}_{5}$, or $\mathrm{AcOH}$ under microwave and in ionic liquids ${ }^{7}$ have been utilized for the condensation reaction. Most recently, this condensation has been reported also to proceed in the presence of CAN, (bromodimethyl)sulfonium bromide, organic acids, and $\mathrm{AgNO}_{3}{ }^{8}$. However, all of these methods have problems such as drastic reaction conditions and several side-reactions. Surface-mediated solid phase reactions are of growing interest ${ }^{9}$ because of their ease of execution and work-up, 
mild reaction conditions, rate of reaction, selectivity, high yields, lack of solvents and low cost in comparison with their homogeneous counterparts. As a part of our efforts to explore the utility of surface-mediated reactions, ${ }^{10-12}$ we report here a new method for the preparation of 1 , 5benzodiazepine derivatives with $\beta$-diketones and $\beta$-ketoesters. It was found that a mixture of $p$ toluenesulfonic acid/celite under solvent-free conditions was capable of producing high yields of 1, 5-benzodiazepines 5a-e by condensation of $o$-phenylenediamine with dicarbonyl compounds 4a-e under mild conditions.

We are interested in the synthesis pyrazolo [4, 3-d] pyrimidin-7-one containing 1, 5benzodiazepines due to the importance of this class of compound in medicinal chemistry. Substituted pyrazolopyrimidinones are potent and selective inhibitors of type 5 cyclic gaunosine3', 5'-monophosphate phosphodiesterase (cGMP) PDE-5 ${ }^{13,14}$ and, as such, have utility in the treatment of male erectile dysfunction (MED) ${ }^{15}$ and female sexual dysfunction (FSD). They also have found use in the treatment of male sexual impotence, with reduced side effects ${ }^{16}$. Substituted pyrazolopyrimidinones are useful also as CNS stimulant, bronchodilator, cardiotonic $^{17}$, herbicide ${ }^{18}$ and antiviral ${ }^{19}$ agents.

\section{Results and Discussion}

As an avenue to such species, 5-(2-ethoxyphenyl)-1-methyl-3-propyl-1,6-dihydro-7H-pyrazolo [4,3-d]pyrimidin-7-one (1) was chlorinated with $\mathrm{POCl}_{3}$ to afford the chlorinated compound 2. The latter was condensed with different $\beta$-diketones/ $\beta$-ketoesters 3a-e in the presence of sodium methoxide to yield $\beta$-diketones/ $\beta$-ketoesters 4a-e (Scheme 1). The condensation of the newly synthesized $\beta$-diketones/ $\beta$-ketoesters 4a-e with $o$-phenylenediamine (o-PDA) in the presence of $p$-toluenesulfonic acid/celite under solvent free conditions, afforeded 3H-1,5-benzodiazepines 5a-e (Scheme 2). 


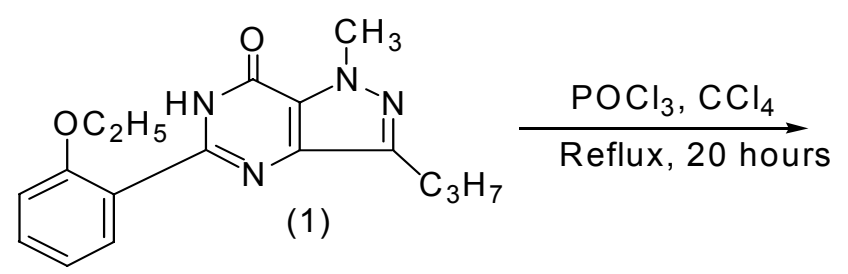

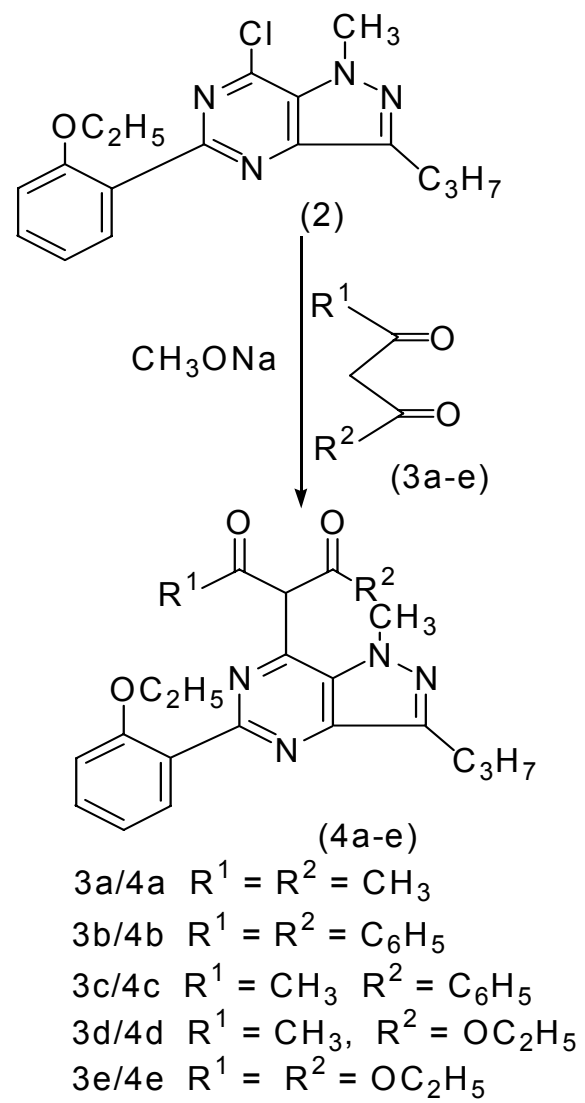

\section{Scheme 1}<smiles>[R]C(=O)C(C([R])=O)c1nc(-c2ccccc2OCC)nc2c(CCCCC)nn(C)c12</smiles><smiles>Nc1ccccc1N</smiles>

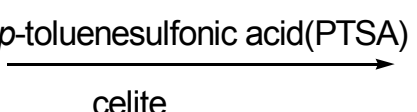
celite

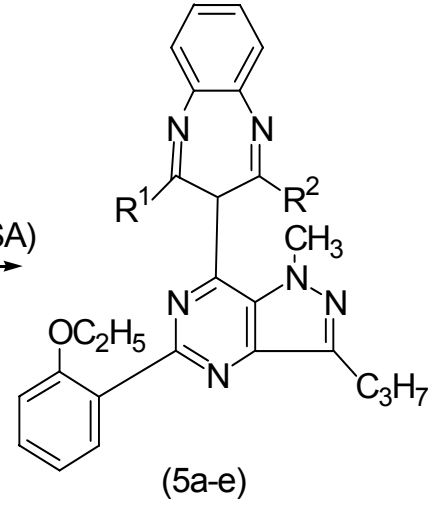

5a $\mathrm{R}^{1}=\mathrm{R}^{2}=\mathrm{CH}_{3}$ $5 b \mathrm{R}^{1}=\mathrm{R}^{2}=\mathrm{C}_{6} \mathrm{H}_{5}$ $5 c R^{1}=\mathrm{CH}_{3} \mathrm{R}^{2}=\mathrm{C}_{6} \mathrm{H}_{5}$ $5 \mathrm{~d} \mathrm{R}^{1}=\mathrm{CH}_{3}, \mathrm{R}^{2}==\mathrm{O}$ 5e $R^{1}=R^{2}==0$

\section{Scheme 2}




\section{Antimicrobial and anthelmintic activities of compounds 5a-e}

The newly synthesized benzodiazepine compounds 5a-e have been screened for antibacterial activity against Staphylococcus aureus and Klebsiella pneumoniae and antifungal activity against Aspergillus niger and Candida albicans by the cup-plate method ${ }^{20,21}$. Crofloxin and Ciclopiroxolamine were used as standards for comparison of antibacterial and antifungal activities, respectively. The results indicate that these compounds were active against all the four organisms. The anthelmintic activity was carried out on earthworms Pherituma posthuma by a technique as described by Bagavant et al. ${ }^{22}$ with slight modification. Piperazine citrate was used as the standard drug. The results of antimicrobial and anthelmintic activity are reported in Table 1 .

Table 1. Antimicrobial and anthelmintic activities of compounds 5a-e

\begin{tabular}{|c|c|c|c|c|c|c|}
\hline \multirow[t]{2}{*}{ Compd. } & \multicolumn{2}{|c|}{$\begin{array}{l}\text { Antibacterial activity zone } \\
\text { of inhibition (in } \mathrm{mm} \text { ) }\end{array}$} & \multicolumn{2}{|c|}{$\begin{array}{l}\text { Antifungal activity zone } \\
\text { of inhibition (in } \mathrm{mm} \text { ) }\end{array}$} & \multicolumn{2}{|c|}{$\begin{array}{l}\text { Anthelmintic } \\
\text { activity (in min.) }\end{array}$} \\
\hline & $\begin{array}{c}\text { A. } \\
\text { aureus }\end{array}$ & K.pneumoniae & A. niger & $\begin{array}{c}\text { C. } \\
\text { albicans }\end{array}$ & Paralysis & $\begin{array}{c}\text { Deat } \\
\mathrm{h}\end{array}$ \\
\hline $5 a$ & 11 & 10 & 15 & 18 & 95 & 90 \\
\hline $5 b$ & 10 & 10 & 16 & 17 & 90 & 120 \\
\hline $5 \mathrm{c}$ & 21 & 24 & 25 & 28 & 105 & 120 \\
\hline $5 d$ & 12 & 15 & 15 & 11 & 90 & 110 \\
\hline $5 \mathrm{e}$ & 14 & 09 & 10 & 19 & 85 & 105 \\
\hline
\end{tabular}

\section{Experimental Section}

General Procedures. All the melting points are uncorrected. The IR spectra were recorded on a Nicolet-Megna-FT-IR-550 spectrometer in KBr pellets. ${ }^{1} \mathrm{H}$ NMR and ${ }^{13} \mathrm{C}$ NMR spectra were run on model DRX 300 at $300.13 \mathrm{MHz}$ in $\mathrm{CDCl}_{3}$ using TMS as internal standard. The purity of the newly synthesized compounds was checked by TLC.

\section{Synthesis of 5-[2-ethoxyphenyl]-1-methyl-7-chloro-1H-pyrazolo [4,3-d) pyrimidine (2)}

To 5-[2-ethoxyphenyl]-1-methyl-3-propyl-1,6-dihydro-7H-pyrimidin-7-one $(3.12 \mathrm{~g}, 0.01 \mathrm{~mol})$ in $\mathrm{CCl}_{4}(50 \mathrm{~mL})$ in a two neck round bottom flask, was added drop wise $\mathrm{POCl}_{3}(10 \mathrm{~mL}$, excess $)$ at $0^{\circ} \mathrm{C}$. After complete addition, the reaction mixture was heated on a water bath for 20 hours. The reaction was monitored through TLC. After completion of the reaction, unreacted $\mathrm{POCl}_{3}$ was removed under reduced pressure. The reaction mass was poured into ice and was extracted with chloroform $(2 \mathrm{x} 50 \mathrm{~mL})$. The chloroform layer was separated and evaporated, to yield a colourless solid. The product was purified by column chromatography on silica gel using pet ether: ethyl acetate (50:50) as eluent and crystallized from methanol. Purity of compound was checked 
through TLC using ethyl acetate: acetone (8:2) system as the mobile phase; mp $165^{\circ} \mathrm{C}$, yield $2.5 \mathrm{~g}, 70 \%$.

Synthesis of 3-[5-(2-ethoxyphenyl)-1-methyl-3-propyl-1H-pyrazolo[4,3-d] pyrimidin-7-yl-]1,3-dimethyl1,3-diphenyl/1-phenyl,3-methyl/1-methyl,3-ethoxy/ 1,3-diethoxy propane-1,3dione (4a-e)

Sodium methoxide $(0.54 \mathrm{~g}, 0.01 \mathrm{~mol})$ and $\beta$-diketones $/ \beta$-ketoesters 3a-e $(0.01 \mathrm{~mol})$ were placed in a dry round bottom flask and stirred for one hour on a magnetic stirrer at a temperature of $50^{\circ} \mathrm{C}$ and a creamy mass was obtained. The chloride derivative $2(3.19 \mathrm{~g}, 0.01 \mathrm{~mol})$ was then added, followed by dry toluene as solvent to effect proper stirring of the reaction mass. The reaction mixture was heated for six hours at $80^{\circ} \mathrm{C}$ with stirring. The progress of the reaction was monitored through TLC. After reaction was complete, the reaction mixture was cooled and toluene was removed. The reaction mixture was extracted using chloroform $(2 \times 50 \mathrm{~mL})$ and washed with water as to remove the salt. The chloroform layer was dried using anhydrous sodium sulphate. Chloroform was evaporated to get solid compound. The product was purified by column chromatography on silica gel using pet. ether: ethyl acetate (50:50) as eluent and crystallized with ethyl acetate and acetone mixture. Purity of the compound was checked through TLC using 7:2:1 (benzene: ethanol: ammonia) upper layer as the mobile phase.

\section{Synthesis of $3 \mathbf{H}$-1, 5-benzodiazepines 5a-e: General procedure}

1,3-Diketones/1,3-ketoesters 4a-e $(0.01 \mathrm{~mol})$ along with $\mathrm{p}$-toluenesulfonic acid/celite (prepared by adding $p$-toluenesulfonic acid ( $1 \mathrm{~g})$ and celite $(1 \mathrm{~g})$ in acetone, stirred for $0.5 \mathrm{~h}$ on magnetic stirrer and then acetone removed by vacuum) mixed in a mortar for 10 minutes, To the aforesaid mixture was added $o$-phenylenediamine $(1.08 \mathrm{~g}, 0.01 \mathrm{~mol})$, heated on a water bath at $60{ }^{0} \mathrm{C}$ for 30 minutes. The reaction mixture was extracted with dichloromethane $(2 \times 100 \mathrm{~mL})$, dried over $\left(\mathrm{Na}_{2} \mathrm{SO}_{4}\right)$, and the solvent was evaporated to give the crude products. The crude products were washed with ether to remove unreacted dicarbonyl compounds. The crude product was recrystallized from pet. ether: ethyl acetate (1:1). Purity of the compound was checked through TLC using 7:2:1 (benzene: ethanol: ammonia) upper layer as the mobile phase. Spectral data for selected products 5a-e.

3-[5-(2-ethoxyphenyl)-1-methyl-3-propyl-1H-pyrazolo[4, 3-d] pyrimidin-7-yl]-2, 4dimethyl-3H-1, 5-benzodiazepine (5a). M.p. $183^{\circ} \mathrm{C}$; yield $85.7 \%$; Anal. Calcd. $\mathrm{C}_{27} \mathrm{H}_{30} \mathrm{~N}_{6} \mathrm{O}$ : C, 71.37; H, 6.61; N, 18.50. Found: C, 71.26; H, 6.60; N, 18.49. IR (KBr): 3060, 2945, 1590, 1480, 1250, $1020 \mathrm{~cm}^{-1} ;{ }^{1} \mathrm{H}$ NMR $\left(300 \mathrm{MHz}, \mathrm{CDCl}_{3}\right): \delta 1.03(\mathrm{t}, J=8.46 \mathrm{~Hz}, 3 \mathrm{H}), 1.60(\mathrm{~m}, J=7.25$ $\mathrm{Hz}, 2 \mathrm{H}), 1.64(\mathrm{t}, J=6.98 \mathrm{~Hz}, 3 \mathrm{H}), 2.30(\mathrm{~s}, 6 \mathrm{H}), 2.55(\mathrm{t}, J=8.34 \mathrm{~Hz}, 2 \mathrm{H}), 3.80(\mathrm{~s}, 3 \mathrm{H}), 4.40(\mathrm{q}, J$ $=8.12 \mathrm{~Hz}, 2 \mathrm{H}), 6.05(\mathrm{~s}, 1 \mathrm{H}), 7.4-8.0(\mathrm{~m}, J=7.36 \mathrm{~Hz}, 8 \mathrm{H}) ;{ }^{13} \mathrm{C} \mathrm{NMR}\left(300 \mathrm{MHz}, \mathrm{CDCl}_{3}\right)$ : $\delta 162.56,149.94,132-128,75.32,67.85,53.20,40.37,29.26,15.85,14.70 ;$ LCMS: 455.5671 $\left(\mathrm{M}+\mathrm{H}^{+}\right)$.

3-[5-(2-ethoxyphenyl)-1-methyl-3-propyl-1H-pyrazolo[4,3-d]pyrimidin-7-yl]-2,4-diphenyl3H-1,5-benzodiazepine (5b). M.p. $219^{\circ} \mathrm{C}$; yield 86.3\%; Anal. Calcd. $\mathrm{C}_{37} \mathrm{H}_{34} \mathrm{~N}_{6} \mathrm{O}$ : C, 77.35; H, 6.09; N, 14.63. Found: C, 76.99; H, 6.10; N, 14.59. IR (KBr): 3040, 2900, 1585, 1492, 1240, 
$1010 \mathrm{~cm}^{-1} ;{ }^{1} \mathrm{H} \mathrm{NMR}\left(300 \mathrm{MHz}, \mathrm{CDCl}_{3}\right): \delta 1.03(\mathrm{t}, J=8.45 \mathrm{~Hz}, 3 \mathrm{H}), 1.65(\mathrm{~m}, J=7.86 \mathrm{~Hz}, 2 \mathrm{H})$, $2.55(\mathrm{t}, J=7.46 \mathrm{~Hz}, 2 \mathrm{H}), 1.65(\mathrm{t}, J=6.95 \mathrm{~Hz}, 3 \mathrm{H}), 4.42(\mathrm{q}, J=8.75 \mathrm{~Hz}, 2 \mathrm{H}), 3.80(\mathrm{~s}, 3 \mathrm{H}), 6.0$ (s, $1 \mathrm{H}), 7.6-8.1(\mathrm{~m}, J=7.66 \mathrm{~Hz}, 18 \mathrm{H}) ;{ }^{13} \mathrm{C} \mathrm{NMR}\left(300 \mathrm{MHz}, \mathrm{CDCl}_{3}\right): \delta 157.50,148.95,132-127$, 76.37, 66.36, 54.20, 41.25, 29.50, 15.84, 14.64; LCMS: 579.7059(M+H $\left.{ }^{+}\right)$.

3-[5-(2-ethoxyphenyl)-1-methyl-3-propyl-1 H-pyrazolo[4,3-d]pyrimidin-7-yl-2-methyl-4-

phenyl-3H-1,5-benzodiazepine (5c). M.p. $211^{\circ} \mathrm{C}$; yield $83.7 \%$ Anal. Calcd. $\mathrm{C}_{32} \mathrm{H}_{32} \mathrm{~N}_{6} \mathrm{O}$ : C, 74.41;H, 6.02; N, 16.27. Found: C, 74.31; H, 6.15; N, 16.10. IR(KBr):3050, 2930, 1585, 1260, $1040 \mathrm{~cm}^{-1}$; ${ }^{1} \mathrm{HNMR}(300 \mathrm{MHz}, \mathrm{CDCl} 3): \delta 1.03(\mathrm{t}, J=8.32 \mathrm{~Hz}, 3 \mathrm{H}), 1.65(\mathrm{~m}, J=7.85 \mathrm{~Hz}, 2 \mathrm{H})$, $1.69(\mathrm{t}, J=9.05 \mathrm{~Hz}, 3 \mathrm{H}), 2.55(\mathrm{t}, J=7.65 \mathrm{~Hz}, 2 \mathrm{H}), 3.80(\mathrm{~s}, 3 \mathrm{H}), 4.42(\mathrm{q}, J=8.75 \mathrm{~Hz}, 2 \mathrm{H}), 6.0$ $(\mathrm{s}, 1 \mathrm{H}), 2.35(\mathrm{~s}, 3 \mathrm{H}), 7.6-8.1(\mathrm{~m}, J=7.95 \mathrm{~Hz}, 13 \mathrm{H}) ;{ }^{13} \mathrm{C} \mathrm{NMR}\left(300 \mathrm{MHz}, \mathrm{CDCl}_{3}\right): \delta 157.23$, 149.90,132-128, 75.34, 68.36, 54.03, 40.32, 29.54, 15.84, 14.66 ; LCMS: 517.6365(M+H $\left.{ }^{+}\right)$.

3-[5-(2-ethoxyphenyl)\}-1-methyl-3-propyl-1H-pyrazolo[4,3-d]pyrimidin-7-yl]-4-methyl-1,3dihydro-3H-1,5-benzodiazepine-2-one (5d). M.p. $189^{\circ} \mathrm{C}$; yield $82.3 \%$; Anal. Calcd. $\mathrm{C}_{26} \mathrm{H}_{28} \mathrm{~N}_{6} \mathrm{O}_{2}$ : C, 68.12; H, 6.11; N, 18.34. Found: C, 68.10; H, 6.03; N, 18.29. IR (KBr):3040, 2900, 1580, 1490, 1260, $1045 \mathrm{~cm}^{-1},{ }_{1} \mathrm{H} \mathrm{NMR}\left(300 \mathrm{MHz}, \mathrm{CDCl}_{3}\right): \delta 1.03(\mathrm{t}, J=8.37 \mathrm{~Hz}, 3 \mathrm{H})$, $1.60(\mathrm{t}, J=8.23 \mathrm{~Hz}, 3 \mathrm{H}), 1.65(\mathrm{~m}, J=8.15 \mathrm{~Hz}, 2 \mathrm{H}), 2.55(\mathrm{t}, J=7.95 \mathrm{~Hz}, 2 \mathrm{H}), 2.50(\mathrm{~s}, 3 \mathrm{H}), 3.80$ $(\mathrm{s}, 3 \mathrm{H}), 4.44(\mathrm{q}, J=7.65 \mathrm{~Hz}, 2 \mathrm{H}), 5.8(\mathrm{~s}, 1 \mathrm{H}), 7.3-8.0(\mathrm{~m}, J=7.65 \mathrm{~Hz}, 4 \mathrm{H}), 8.75(\mathrm{~s}, 1 \mathrm{H}) ;{ }^{13} \mathrm{C}$ NMR (300MHz, $\left.\mathrm{CDCl}_{3}\right): \delta 165.34,158.454,149.12,134-126,75.85,68.34,54.37,40.35,28.60$, 15.74, 14.47; LCMS: 457.5399( $\left.\mathrm{M}+\mathrm{H}^{+}\right)$.

3-[5-(2-ethoxyphenyl)\}-1-methyl-3-propyl-1H-pyrazolo[4,3-d]pyrimidin-7-yl]-1,5-dihydro3H-1,5-benzodiazepine-2,4-dione (5e). M.p. $203^{\circ} \mathrm{C}$; yield $85.4 \%$; Anal. Calcd. $\mathrm{C}_{25} \mathrm{H}_{26} \mathrm{~N}_{6} \mathrm{O}_{2}$ : C, 65.50; H, 5.69; N, 18.34. Found: C, 65.49; H, 5.61; N, 8.17. IR (KBr): 3050, 2920, 1560, 1490, 1250, $1030 \mathrm{~cm}^{-1} ;{ }^{1} \mathrm{H}$ NMR $\left(300 \mathrm{MHz}, \mathrm{CDCl}_{3}\right): \delta 1.02(\mathrm{t}, J=8.35 \mathrm{~Hz}, 3 \mathrm{H}), 1.64(\mathrm{t}, J=7.65 \mathrm{~Hz}$, $1.70(\mathrm{~m}, J=7.95 \mathrm{~Hz}, 2 \mathrm{H}), 2.55(\mathrm{t}, J=6.98 \mathrm{~Hz}, 2 \mathrm{H}), 3.80(\mathrm{~s}, 3 \mathrm{H}), 4.40(\mathrm{q}, J=7.35 \mathrm{~Hz}, 2 \mathrm{H}), 6.10$ $(\mathrm{s}, 1 \mathrm{H}), 7.5-8.0(\mathrm{~m}, J=7.85 \mathrm{~Hz}, 8 \mathrm{H}), 8.65 \mathrm{~s}, 2 \mathrm{H}) ;{ }^{13} \mathrm{C} \mathrm{NMR}\left(300 \mathrm{MHz}, \mathrm{CDCl}_{3}\right): \delta 165.5,157.56$,

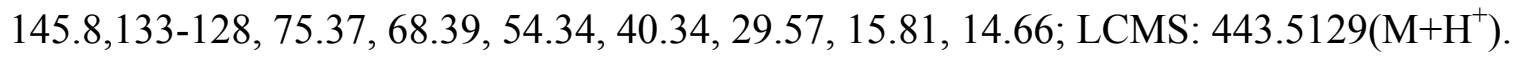

\section{Acknowledgements}

The authors are thankful to the Head, Department of Chemistry, University of Rajasthan, Jaipur for providing laboratory facilities. The authors are thankful also to Central Drug Research Institute, Lucknow for providing spectral data. One of them (Rajesh Kumar) is thankful to the UGC for the award of Senior Research Fellowship. 


\section{References}

1. Schutz, H. Benzodiazepines; Springer: Heidelberg, 1982. (b) Landquist, J. K. In Comprehensive Heterocyclic Chemistry; Katritzky, A. R.; Rees, C. W. Pergamon: Oxford, 1984, Vol. 1, pp166-170. (c) Fryer, R. I. Bicyclic Diazepines, In Comprehensive Heterocyclic Chemistry; Taylor, E. C.; Ed.; Wiley: New York, 1991; Vol. 50, Chapter II. (d) Randall, L. O.; Kappel, B. In Benzodiazepines; Garattini, S.; Musini, E.; Randall, L. O. Eds.; Raven Press: New York, 1973; p 27.

2. De Baun, J. R.; Pallos, F. M.; Baker, D. R. U.S. Patent 3, 978, 227, 1976; Chem. Abstr. 1977, 86, 5498d.

3. Harris, R. C.; Straley, J. M. U.S. Patent 1, 537,757, 1968; Chem. Abstr. 1970, 73, 100054w.

4. Aversa, M. C.; Ferlazzo, A.; Gionnetto, P.; Kohnke, F. H. Synthesis 1986, 230. (b) Essaber, M.; Hasnaoui, A.; Benharref, A.; Lavergne, J. P. Synth. Commun. 1998, 28, 4097. (c) EISayed, A. M.; Abdel-Ghany, H.; EI-Saghier, A. M. M. Synth Commun. 1999, 29, 3561. (d) Chimirri, A.; Grasso, S.; Ottana, R.; Romeo, G.; Zappala, M. J. Heterocycl. Chem. 1990, 27,371 .

5. Stahlhofen, P.; Ried, W. Chem. Ber. 1957, 90, 815.

6. Ried, W.; Torinus, E. Chem. Ber. 1959, 92, 2902.

7. (a) Balakrishna, M. S.; Kaboudin, B. Tetrahedron Lett. 2001, 42, 1127. (b) Curini, M.; Epifano, F.; Marcotullio, M. C.; Rosati, O. Tetrahedron Lett. 2001, 42, 3197. (c) Sabita, G.; Reddy, G. S. K.; Reddy, K. B.; Reddy, N. M.; Yadav, J. S. Adv. Synth.Catal. 2004, 346, 921. (d) Kaboudin, B.; Naveen, K. Heterocycles 2001, 55, 1443. (e) Pozaretzi, M.; Stephanatou, J. S.; Tsoleridis, C. A.; Tetrahedron Lett, 2002, 43, 1755. (f) Reddy, B. M.; Sreekant, P. M.; Tetrahedron Lett. 2003, 44, 4447. (g) Yadav, J. S.; Reddy, B. V. S.; Eshwaraiah, B.; Auradha, K. Green Chem. 2002, 4, 592. (h) Jarikote, D. V.; Siddiqui, S. A.; Rajagapol, R.; Daniel, T.; Lahoti, R. J .; Srinivasan, K V. Tetrahedron Lett. 2003, 44, 1835. (i) De, S. K.; Gibbs, R. A. Tetrahedron Lett. 2005, 46, 1811.

8. Yadav, J. S.; Reddy, B. V. S.; Praveen kumar, S.; Nagaiah, K. Synthesis, 2005, 480. (b) Das, B.; Ramu, R.; Ravikanth, B.; Reddy, V. S. J. Mol. Catal. A Chem. 2006, 76, 246.(c) Thakuria, H.; Pramanik, A.; Borah, B. M.; Das, G. Tetrahedron Lett. 2006, 47, 3135. (d) Varala, R.; Enugala, R.; Nuvula, S.; Adapa, S. R. Synlett. 2006, 1009. (d) Kumar, R.; Chaudhary, P.; Nimesh, S.; Verma, A. K.; Chandra, R. Green Chem. 2006, 8, 519.

9. Fadel, A.; Yefash, R.; Saluan, J. Synthesis 1987, 37. (b) Rosini, G.; Galarini, R.; Marotta, E.; Righi, R. J. Org. Chem. 1990, 55, 781. (c) Kodomari, M.; Sakamoto, T.; Yoshitomi, S. J. Chem. Soc., Chem. Commun. 1990, 701. (d) Kropp, P. J.; Duas, K. A.; Crawford, S. D.; Tubergren, M. W.; Kepler, K. D.; Craig S L \& Wilson, V P, J. Am. Chem. Soc., 1990, 112, 7433. (e) Hondrogiannis, G.; Pagni, R. M.; Kabalka, G. W.; Anisoki, P.; Kurt, R. Tetrahedron Lett. 1990, 31, 5433. (f) Pantney, H. K. Tetrahedron Lett. 1991, 32, 2259. (g) Pauter, F.; Daudon, M. Tetrahedron Lett. 1991, 32, 1457

10. Sardarian, A. R.; Kaboudin, B. Synth. Commun. 1997, 27, 543. 
11. Sardarian, A. R.; Kaboudin, B. Tetrahedron Lett. 1997, 38, 2543.

12. Kaboudin, B. J. Chem. Rev. 1999, 402.

13. Bunnage, M. K.; Mathias, J. P.; Street, S. D. A.; Wood, A. PCT Int. Appl. WO 9954, 333, (ClCO7D, 487/14), 28 Oct, 1999, G.B. Appl. 1998/14, 187, 30 Jun. 1998; 221pp; Chem. Abstr. 1999, 131, 310644f.

14. Bell, A. S.; Terrett, N. K. Eur. Pat. EP 526, 004 (Cl. CA7D, 487/04), 03 Feb. 1993, GB Appl. 01/14, 760, 9 Jul 1991, 34 pp: Chem. Abstr. 1993, 118, 254957c.

15. Doherty, P. C.; Place, V. A.; Jr. and Smith W. L. PCT Int. Appl. Wo 9921, 558 (Cl. A61K 31/485) 6 May 1999, US Appl. 181, 070, 27 Oct. 1998, 31 pp: Chem. Abstr. 1999, 130, 332908d.

16. Yoo, M.; Kim, W.; Chang, M. S.; Lim, J. I.; Kim, D. S.; Kim I Y, Lim T K, Ann B O, Kang K K, Son M, Doh H, Kim S, Shim H, Oh T, Kim H and Kim D G, PCT Int. Appl. Wo 0027, 848 (Cl. CO7D, 487/14) 18 May 2000, KR Appl. 9, 949, 9 Nov. 1999: Chem. Abstr. 2000, $132,334469 \mathrm{z}$.

17. Warner-Lambert Co., Jpn. Kokai Tokkyo Koho Jp61, 236, 778 [86, 236, 778] (Cl. CO7D 87/010, 22 Oct. 1986, US Appl. 720, 437, 05 Apr. 1985: Chem. Abstr. 1987, 106, 101967h.

18. Lindell, S. D.; Moloney, B. A.; Hewitt, B.D.; Earnhaw, C. G.; Philip, P. J.; Dancer, J. E. Bioorg. Med. Chem. Lett. 1999, 9, 1985.

19. Sanghvi, Y. S.; Larson, S. B.; Smee, D. F.; Revankar, G. R.; Robins, R. K. Nucleosides Nucleotides 1991, 10, 1417.

20. Sundane, A. R.; Rudresh, K.; Satynarayan, N. D.; Hiremath, S. P. Indian J. Pharm. Sci. 1989, 60, 379.

21. Giand, K. N.; Dar, R. N.; Chopra, B. M.; Kaul, R. N. Indian J. Pharm. Sci. 1965, 27, 141.

22. Bagvant, G.; Gole, S. R.; Joshi, V.; Soni, S. B. Indian J. Pharm. Sci. 1994, 56, 80. 